1999-11-22

\title{
Simulation and density functional study of a simple membrane separating two restricted primitive model electrolytes
}

\author{
Richard L. Rowley \\ rowley@byu.edu \\ Dezso Boda \\ Douglas Henderson \\ Stefan Sokolowski
}

Follow this and additional works at: https://scholarsarchive.byu.edu/facpub

Part of the Chemical Engineering Commons

\section{Original Publication Citation}

D. Boda, D. Henderson, R. Rowley, and S. Sokolowski, â€œSimulation and density functional study of a simple membrane separating two restricted primitive model electrolytesâ€, J. Chem. Phys.111, 9382 (1999)

\section{BYU ScholarsArchive Citation}

Rowley, Richard L.; Boda, Dezso; Henderson, Douglas; and Sokolowski, Stefan, "Simulation and density functional study of a simple membrane separating two restricted primitive model electrolytes" (1999). Faculty Publications. 608.

https://scholarsarchive.byu.edu/facpub/608 


\title{
Simulation and density functional study of a simple membrane separating two restricted primitive model electrolytes
}

\author{
Dezső Boda a) and Douglas Henderson ${ }^{\text {b) }}$ \\ Department of Chemistry and Biochemistry, Brigham Young University, Provo, Utah 84602-5700 \\ Richard Rowleyc) \\ Department of Chemical Engineering, Brigham Young University, Provo, Utah 84602-5700 \\ Stefan Sokołowskid) \\ Department for Modelling Physico-Chemical Processes, MCS University, 20031 Lublin, Poland
}

(Received 9 July 1999; accepted 25 August 1999)

\begin{abstract}
A simple membrane, supporting charge densities $\sigma_{1}$ and $\sigma_{2}=-\sigma_{1}$ on its inner and outer surfaces, is considered. In addition to the electrostatic potential, the membrane interacts with the surrounding fluid by a short range van der Waals-like potential. The fluid beyond the outer surface is a three-component restricted primitive electrolyte consisting of two cations and one anion. The membrane is impermeable to one of the cations so that the fluid in the membrane and beyond the inner surface is a two-component restricted primitive electrolyte. We use Monte Carlo simulations and density functional theory to study the density profiles of the electrolyte and the charge-electrostatic potential relationship for the membrane surfaces. Even though $\sigma_{2}=-\sigma_{1}$, the potentials on the membrane surfaces are not equal and opposite. We also study a membrane consisting of a single charged plane. For both models, the density functional results are in good agreement with the simulations. (C) 1999 American Institute of Physics. [S0021-9606(99)51243-9]
\end{abstract}

\section{INTRODUCTION}

Membranes are of interest in biological and chemical engineering processes. Biological membranes are responsible for the ability of the roots of plants to receive nutrients from their environment. Supported liquid membranes can be used for the selective and efficient extraction and enrichment of analytes prior to analysis.

Theories of membranes and fluids near membranes have been developed by several authors. ${ }^{1-7}$ Much of the past work has been based on the assumption of zero membrane thickness. Obviously, this is an idealization.

In most experimental work, the membrane thickness plays an important role. ${ }^{7-9}$ The model of Ellin ${ }^{10}$ for neurons is an electrolyte solution that is bounded by a membrane of finite thickness. The membrane is modeled as a dielectric continuum with a uniform surface charge density on each of the membrane surfaces.

Many studies of electrolytes near membranes are undertaken at the level of the Poisson-Boltzmann (PB) theory. The PB theory results from a combination of Poisson's equation, which is exact, with a simple Boltzmann function representing the structure of the electrolyte. It is the Boltzmann function that is approximate. In the PB theory, the ions are modeled as point ions or, at most, the size of the ions is introduced in an asymmetric manner. Further, the molecular

\footnotetext{
${ }^{a)}$ Permanent address: Department of Physical Chemistry, University of Veszprém, H-8201 Veszprém, P.O. Box 158, Hungary; electronic mail: boda@almos.vein.hu

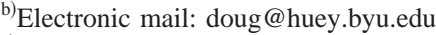

c)Electronic mail: rowley@byu.edu

${ }^{d)}$ Electronic mail: stefan@ paco.umcs.lublin.pl
}

nature of the solvent is ignored. The solvent is represented by a dielectric continuum whose dielectric constant is equal to that of the solvent. Generally speaking, the PB theory is most appropriate for low electrolyte concentrations.

To our knowledge, molecular models of the solvent have not been considered. Molecular models of the solvent have led to an understanding of important effects in other contexts. ${ }^{11,12}$ There is no reason to believe that the assumption of a continuum solvent is any more valid for a membrane than in the other problems.

In this paper we formulate a simple model for a solvent. The electrolyte is represented by a mixture of charged hard spheres of charge $q_{i}$ and of equal size, $d$, and the solvent is represented by a continuum of dielectric constant $\epsilon$. This model is called the restricted primitive model (RPM). The effect of the molecular nature of the solvent will be explored in subsequent papers.

We employ two techniques in our study. The first is computer simulations. Our method is based on that of Torrie and Valleau. ${ }^{13}$ The second method is density functional theory. We use a version of the Rosenfeld theory, ${ }^{14,15}$ modified and simplified by Kierlik and Rosinberg. ${ }^{16,17}$ Other versions, not used here, have been developed for inhomogeneous electrolytes. ${ }^{18,19}$

\section{MODEL AND THEORY}

We consider a pair of membranes of thickness $\Delta$ centered at $z=0$ and separated by a distance $L$. The linear distance is denoted by $z$. The symmetry about $z=0$ is assured. In this paper we assume $L$ is very large so that the two membranes are noninteracting. The inner and outer surfaces 

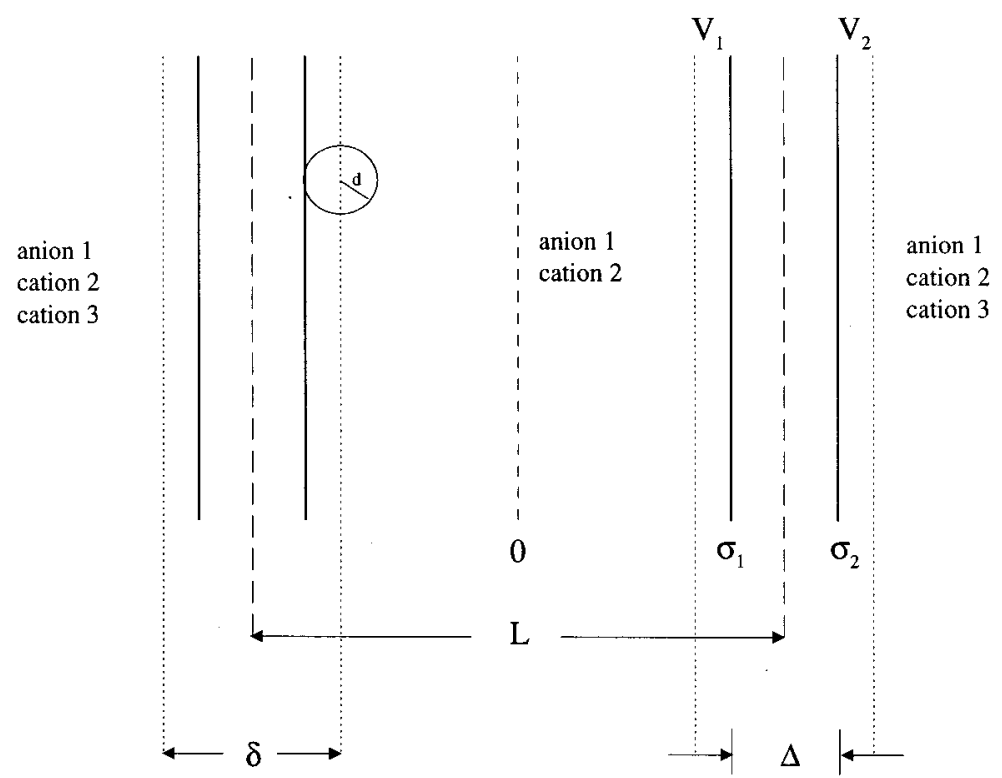

FIG. 1. Geometry used in the calculations.

of each membrane are given the charge densities $\sigma_{1}$ and $\sigma_{2}$, respectively. The corresponding electric potentials are $V_{1}$ and $V_{2}$. In addition, a short range van der Waals force that acts upon the ions and that extends a short distance beyond the membrane surfaces is assumed. We represent this force with a square well potential. The geometry of the system that we consider is displayed in Fig. 1. For instance, this geometry might correspond to the cross section of a biological cell with $z=0$ being the center of the interior of the cell.

The interaction between the ions is

$$
u_{i j}(r)=\left\{\begin{array}{l}
\infty, \quad r<d \\
\frac{q_{i} q_{j}}{\epsilon r}, \quad r>d
\end{array}\right.
$$

where $q_{i}$ is the charge of an ion of species $i$ and $r$ is the separation of the ions. The electrolyte outside the membrane has three components, an anion of charge $-q$ and two cations, both of charge $q$. The quantity $\epsilon$ is the dielectric constant, which we assume is uniform throughout the entire system and the same for all distances.

The membrane is presumed to be impermeable to the cations of species 3. Therefore, the electrolyte inside the membrane consists of the components of charge $\pm q$. Far from the membrane, the charge neutrality exists. Thus,

$$
\rho_{1}=\rho_{2}+\rho_{3},
$$

for $z \rightarrow \infty$ and

$$
\rho_{1}=\rho_{2},
$$

for $z \rightarrow 0$.

The interaction between the ions of species $i$ and the membrane is given by

$$
u_{i}(z)=\mathrm{v}_{i}(z)+w_{i}(z),
$$

where $w_{i}(z)$ and $\mathbf{v}_{i}(z)$ are the electrostatic and the nonelectrostatic van der Waals parts of the external potential, respectively. The Boltzmann factor for the van der Waals interaction is

$$
e^{-\beta \vee_{i}(z)}= \begin{cases}B, & \frac{L-\delta}{2}<|z|<\frac{L+\delta}{2} \\ 1, & \text { otherwise }\end{cases}
$$

for the ions of species 1 and 2 . The parameter $B$ limits the access of the ions to the interior of the membrane (or promotes access if $B$ exceeds 1). Two values of $B$ are considered, 0.1 and 0.001 , corresponding, respectively, to a more permeable and less permeable membrane. The Boltzmann factor for the ions of species 3 is

$$
e^{-\beta \vee_{3}(z)}= \begin{cases}0, & |z|<\frac{L-\delta}{2} \\ 1, & \text { otherwise }\end{cases}
$$

Equations (5) and (6) apply for the membranes both at $z=-L / 2$ and $z=L / 2$ due to the symmetry about $z=0$. The width of the van der Waals square well potential is denoted by $\delta$.

The electrostatic interaction between an ion and a surface (or sheet of charge) is given by

$$
w_{i}(z)=\frac{4 \pi \sigma q_{i}}{\epsilon} z^{\prime},
$$

where $\sigma$ is the uniform charge per unit area, and equals $\sigma_{1}$ or $\sigma_{2}$ as appropriate, and $z^{\prime}$ is the distance from the sheet of charge.

$$
\begin{aligned}
& \text { For } z>\frac{L+\Delta}{2}, \\
& \frac{d \Phi(z)}{d z}=\frac{4 \pi}{\epsilon} \sum_{j} q_{j} \rho_{j} \int_{z}^{\infty} g_{j}(t) d t
\end{aligned}
$$

and

$$
\Phi(z)=-\frac{4 \pi}{\epsilon} \sum_{j} q_{j} \rho_{j} \int_{z}^{\infty}(t-z) g_{j}(t) d t,
$$


where $\Phi(z)$ and $g_{j}(z)=\rho_{j}(z) / \rho_{j}$ are the electrostatic potential and the reduced local density of the ions of species $j$, respectively.

$$
\begin{aligned}
& \text { For } 0<z<\frac{L-\Delta}{2}, \\
& \frac{d \Phi(z)}{d z}=-\frac{4 \pi}{\epsilon} \sum_{j} q_{j} \rho_{j} \int_{0}^{z} g_{j}(t) d t .
\end{aligned}
$$

Equation (10) assumes that the two membranes are symmetric so that $d \Phi / d z=0$ at $z=0$. The potential is given by

$$
\Phi(z)=\frac{4 \pi}{\epsilon} \sum_{j} q_{j} \rho_{j} \int_{0}^{z}(t-z) g_{j}(t) d t
$$

Equation (11) assumes that $L$ is large enough that $\Phi(0)$ $=0$. The situation is more complex when $L$ is not large and the density profiles about the two membranes overlap or when the system is not symmetric about $z=0$. These cases do not arise in this paper; they will be discussed in future studies.

The potentials $V_{1}$ and $V_{2}$ are related to the profiles by

$$
\begin{aligned}
V_{2}= & \Phi\left(\frac{L+\Delta}{2}\right)=-\frac{4 \pi}{\epsilon} \sum_{j} q_{j} \rho_{j} \\
& \times \int_{\frac{L+\Delta}{2}}^{\infty}\left(t-\frac{L+\Delta}{2}\right) g_{j}(t) d t
\end{aligned}
$$

and

$$
\begin{aligned}
V_{1} & =\Phi\left(\frac{L-\Delta}{2}\right) \\
& =-\frac{4 \pi}{\epsilon} \sum_{j} q_{j} \rho_{j} \int_{0}^{\frac{L-\Delta}{2}}\left(\frac{L-\Delta}{2}-t\right) g_{j}(t) d t .
\end{aligned}
$$

The region $[(L-\Delta) / 2]<z<[(L+\Delta) / 2]$ is a little more complex. The result is

$$
\frac{d \Phi(z)}{d z}=-\frac{4 \pi}{\epsilon} \sum_{j} q_{j} \rho_{j} \int_{\frac{L-\Delta}{2}}^{z} g_{j}(t) d t+c_{1}
$$

and

$$
\Phi(z)=-\frac{4 \pi}{\epsilon} \sum_{j} q_{j} \rho_{j} \int_{\frac{L-\Delta}{2}}^{z}(z-t) g_{j}(t) d t+c_{1} z+c_{2} .
$$

The constants $c_{1}$ and $c_{2}$ are determined by

$$
V_{1}=\Phi\left(\frac{L-\Delta}{2}\right)
$$

and

$$
V_{2}=\Phi\left(\frac{L+\Delta}{2}\right)
$$

The result is

$$
c_{1}=\frac{V_{2}-V_{1}}{\Delta}+\frac{4 \pi}{\epsilon \Delta} \sum_{j} q_{j} \rho_{j} \int_{\frac{L-\Delta}{2}}^{\frac{L+\Delta}{2}}\left(\frac{L+\Delta}{2}-t\right) g_{j}(t) d t
$$

and

$$
c_{2}=V_{1}-c_{1} \frac{L-\Delta}{2} \text {. }
$$

The charge on each wall can be computed from the charge profiles using Gauss' law. Thus,

$$
\sigma_{1}=-\frac{\epsilon}{4 \pi} c_{1}-\sum_{j} q_{j} \rho_{j} \int_{0}^{\frac{L-\Delta}{2}} g_{j}(t) d t
$$

and

$$
\sigma_{2}=\frac{\epsilon}{4 \pi} c_{1}-\sum_{j} q_{j} \rho_{j} \int_{\frac{L-\Delta}{2}}^{\infty} g_{j}(t) d t .
$$

Overall charge neutrality,

$$
\sigma_{1}+\sigma_{2}=-\sum_{j} q_{j} \rho_{j} \int_{0}^{\infty} g_{j}(t) d t
$$

is satisfied as would be expected. However, as emphasized by Lozada-Cassou, ${ }^{20}$ the charges in the regions $0<z<[(L$ $-\Delta) / 2]$ and $[(L+\Delta) / 2]<z<\infty$ do not equal $\sigma_{1}$ and $\sigma_{2}$, respectively, except under special circumstances or as an approximation. Even setting $V_{1}=V_{2}$ does not yield local charge neutrality unless there is no net charge within the membrane.

It is convenient to introduce reduced or dimensionless units in our calculations. The reduced temperature is

$$
T^{*}=\frac{k \epsilon d}{q^{2}} T,
$$

where $k$ is the Boltzmann constant. The reduced charge densities on the surfaces are

$$
\sigma_{i}^{*}=\frac{\sigma_{i} d}{q}
$$

and the reduced potential is

$$
\Phi *(z)=\beta e \Phi(z),
$$

where $\beta=1 / k T$. All distances are reduced by the core diameter $d$.

In this work we use the value for $T^{*}$ that was chosen by Torrie and Valleau, ${ }^{13}$

$$
1 / T^{*}=1.6808
$$

or, equivalently, a reduced charge of

$$
q^{*}=\frac{1}{\sqrt{T^{*}}}=\sqrt{\frac{\beta q^{2}}{\epsilon d}}=1.296
$$

This corresponds to an aqueous solution at room temperature with the core diameter $d=4.25 \AA$. Here we restricted our attention to

$$
\delta=\Delta+d .
$$

However, we consider two values of $\Delta$. The value of $L$ is unimportant, so long as it is large.

\section{SIMULATIONS}

Monte Carlo simulations have been performed in a canonical ensemble at fixed temperature, particle numbers and volume. A rectangular simulation cell of dimensions $W \times W$ $\times H$ was used with a membrane in the middle. Later we will consider the case of two membranes. The system was con- 
fined between two hard walls that are placed at $z=0$ and $z$ $=H$. The length of the cell was chosen to be $H=24 d$; this is long enough so that the density profiles at the walls and the membranes do not overlap and profiles of neutral bulk electrolytes are obtained in the middle of both halves of the cell.

The number of particles was chosen to give bulk concentrations of about $1 \mathrm{M}$ (this corresponds to a partial reduced density of about 0.046 using $d=4.25 \AA$ ). In this study we consider a system where the densities of penetrating and nonpenetrating cations are nearly the same. Therefore, three times more cations of species 2 (penetrating) than those of species 3 (nonpenetrating cations) were used, i.e., $N_{2}$ $=3 N_{3}$. Charge neutrality was ensured so that $N_{1}=N_{2}$ $+N_{3}$. In the case where the membrane consists of two planes of equal and opposite charge, there is no net charge carried by the membrane. In the case of a membrane consisting of a single charged surface, a compensating charge exists near the outer hard walls.

Besides the displacement of the particles (with a maximum displacement of $0.5 d$ ), another MC move was applied. An attempt to move the penetrating particles from one side of the membrane to the other side at a randomly generated position was made. These "jumps" ensured faster equilibration between the two sides of the membrane. Using only the particle displacement, the ions would have had difficulty in passing through the membrane due to the van der Waals and/or electrostatic repulsions; this would have resulted in slower equilibration and weaker sampling.

Long range corrections were taken into account by the charged sheet method that was described in detail elsewhere. ${ }^{13,21}$ The length of the simulation runs were 4 million cycles; a move for every particle was attempted in each cycle.

\section{DENSITY FUNCTIONAL THEORY}

As has been mentioned earlier, the electrolyte solution is in contact with a semipermeable membrane built of two planes of the surface $S$ with external charges $\sigma_{2}$ and $\sigma_{1}$, on the outside and inside, respectively. When dealing with longranged Coulomb forces, it is convenient to consider the electrostatic contribution to the free energy separately. ${ }^{22}$ According to Kierlik and Rosinberg, ${ }^{16,17}$ we write the system grand potential in the form

$$
\begin{aligned}
\Omega= & G\left(\left\{\rho_{i}\right\}\right)+\frac{1}{2} \sum_{i} \int \rho_{i}(\mathbf{r}) q_{i} \Phi(\mathbf{r}) d \mathbf{r} \\
& +\sum_{i} \int \rho_{i}(\mathbf{r})\left[\mathrm{v}_{i}(\mathbf{r})-\mu_{i}\right] d \mathbf{r},
\end{aligned}
$$

where $\rho_{i}(\mathbf{r})$ are the ionic densities, $\mu_{i}$ are the chemical potentials, $\mathbf{v}_{i}(\mathbf{r})$ is the nonelectrostatic part of the external potential that defines the membrane, $G\left(\left\{\rho_{i}\right\}\right)$ is a (unspecified for the moment) functional of the ionic densities and $\Phi(\mathbf{r})$ is the mean electrostatic potential, related to the ionic densities via Poisson's equation,

$$
\nabla^{2} \Phi(\mathbf{r})=-\frac{4 \pi}{\epsilon} \sum_{i} q_{i} \rho_{i}(\mathbf{r}),
$$

where $\rho_{i}(\mathbf{r})=\rho_{i} g_{i}(\mathbf{r})$.

The approximation $G\left(\left\{\rho_{i}\right\}\right)=F_{\mathrm{HS}}\left(\left\{\rho_{i}\right\}\right), \quad$ where $F_{\mathrm{HS}}\left(\left\{\rho_{i}\right\}\right)$ is the free energy functional of a hard sphere system, would correspond to a mean field treatment of the electrostatic forces, analogous to the Gouy-Chapman theory, ${ }^{23,24}$ except that the hard-sphere effects are now included. To proceed further, we use a functional Taylor expansion of $G\left(\left\{\rho_{i}\right\}\right)$ in powers of $\Delta \rho_{i}(\mathbf{r})=\rho_{i}(\mathbf{r})-\rho_{i b}$, where $\rho_{i b}$ is the bulk density of a homogeneous neutral fluid

$$
\begin{aligned}
& G\left[\left\{\rho_{i}\right\}\right]=F_{\mathrm{id}}\left[\left\{\rho_{i}\right\}\right]+\int g^{\mathrm{ex}}\left(\left\{\rho_{i}\right\}\right) d \mathbf{r} \\
& \quad+\sum_{i}\left(\mu_{i}^{\mathrm{ex}}-q_{i} \Phi_{\text {bulk }}\right) \int \Delta \rho_{i}(\mathbf{r}) d \mathbf{r} \\
& \quad-\frac{k T}{2} \sum_{i, j} \int c_{i j}^{\mathrm{sr}}\left(\left|\mathbf{r}-\mathbf{r}^{\prime}\right|\right) \Delta \rho_{i}(\mathbf{r}) \Delta \rho_{i}\left(\mathbf{r}^{\prime}\right) d \mathbf{r} d \mathbf{r}^{\prime}+\cdots
\end{aligned}
$$

In the above, $c_{i j}^{\mathrm{sr}}$ is the short-ranged part of the direct correlation function

$$
c_{i j}^{\mathrm{sr}}(r)=c_{i j}(r)+\frac{\beta q_{i} q_{j}}{\epsilon r},
$$

where $c_{i j}(r)$ is the direct correlation function of the corresponding uniform fluid at the same bulk density $\left\{\rho_{i b}\right\}$ as the ionic fluid, $F_{\text {id }}\left(\left\{\rho_{i}\right\}\right)$ is the ideal part of the free energy, $\mu_{i}^{\text {ex }}$ is the excess (apart from ideal contribution) parts of the chemical potential, and $g^{\operatorname{ex}}\left(\left\{\rho_{i}\right\}\right)$ is the Helmholtz excess free energy density of the uniform ionic mixture minus the electrostatic energy. The higher order terms [not displayed explicitly in Eq. (31)] contain three- and higher-order direct correlation functions $\left(c_{i j k}^{s r}, c_{i j k l}^{s r}, \ldots\right)$. All these functions can be formally separated to get equations analogous to Eq. (32).

It is possible to sum the hard-sphere contributions to all orders. Doing that, defining $\Delta c_{i j}(r)=c_{i j}^{\mathrm{sr}}(r)-c_{i j}^{\mathrm{HS}}(r)$, and neglecting the corresponding higher order terms $\Delta c_{i j k}(r)$, $\Delta c_{i j k l}(r), \ldots$, we obtain

$$
\begin{aligned}
G\left(\left\{\rho_{i}\right\}\right)= & F_{\mathrm{HS}}\left(\left\{\rho_{i}\right\}\right)+\int\left[g^{\mathrm{ex}}\left(\left\{\rho_{i}\right\}\right)-f_{\mathrm{HS}}^{\mathrm{ex}}\left(\left\{\rho_{i}\right\}\right)\right] d \mathbf{r} \\
& +\sum_{i}\left(\mu_{i}^{\mathrm{ex}}-\mu_{i, \mathrm{HS}}-q_{i} \Phi_{\mathrm{bulk}}\right) \int \Delta \rho_{i}(\mathbf{r}) d \mathbf{r} \\
& +\frac{k T}{2} \int \Delta c_{i j}\left(\left|\mathbf{r}-\mathbf{r}^{\prime}\right|\right) \Delta \rho_{i}(\mathbf{r}) \Delta \rho_{i}\left(\mathbf{r}^{\prime}\right) d \mathbf{r} d \mathbf{r}^{\prime},
\end{aligned}
$$

where $\mu_{i, \mathrm{HS}}$ and $f_{\mathrm{HS}}^{\mathrm{ex}}$ are the chemical potentials and the excess Helmholtz free energy density of the uniform hardsphere fluid, respectively.

The requirement $\delta \Omega / \delta \rho_{i}(r)=0$ leads to the EulerLagrange equation

$$
\frac{\delta G}{\delta \rho_{i}(\mathbf{r})}+q_{i} \Phi(\mathbf{r})+\mathrm{v}_{i}(\mathbf{r})=\mu_{i} .
$$

From Eqs. (33) and (34), we obtain the final equation for the density profile 


$$
\begin{aligned}
-k T & \ln \left[\rho_{i}(\mathbf{r}) / \rho_{i}\right] \\
= & \mathrm{v}_{i}(\mathbf{r})+\left[\frac{\delta F_{\mathrm{HS}}^{\mathrm{ex}}}{\delta \rho_{i}(\mathbf{r})}-\mu_{i, \mathrm{HS}}^{\mathrm{ex}}\right]+q_{i}\left[\Phi(\mathbf{r})-\Phi_{\mathrm{bulk}}\right] \\
& -k T \sum_{j} \int \Delta c_{i j}\left(\left|\mathbf{r}-\mathbf{r}^{\prime}\right|\right) \Delta \rho_{j}\left(\mathbf{r}^{\prime}\right) d \mathbf{r}^{\prime} .
\end{aligned}
$$

For the hard-sphere part, $F_{\mathrm{HS}}^{\mathrm{ex}}$, we adopt one of the most accurate nonlocal functionals, i.e., a version of the Rosenfeld theory ${ }^{14,15}$ modified and simplified by Kierlik and Rosinberg. ${ }^{16,17}$ Because this theory is quite standard, we refer the reader to these papers.

The solution of Eq. (35) requires knowledge of the bulk direct correlation functions, $\Delta c_{i j}(r)$. The most widely used expression is the mean spherical approximation (MSA) result which is of reasonable accuracy and yields analytical expressions for the direct correlation functions. In the case of the primitive model used here, we have $\mathrm{e}^{25}$

$$
k T \Delta c_{i j}(r)=\left\{\begin{array}{l}
-\frac{q_{i} q_{j}}{\epsilon}\left[\frac{2 A}{d}-\left(\frac{A}{d}\right)^{2} r-\frac{1}{r}\right], \quad r<d . \\
0, \quad r>d
\end{array}\right.
$$

In the above expression, $d$ is the ionic diameter, $A$ $=x^{-2}\left[x^{2}+x-x(1+2 x)^{1 / 2}\right]$, and $x=\left(4 \pi \beta d^{2} / \epsilon\right) \sum_{i} q_{i} \rho_{i}$.

The main deficiencies of the MSA theory are well known. ${ }^{26}$ In order to improve the theory, one should use more elaborate theory, for example the generalized MSA or GMSA theory, ${ }^{27}$ or the hypernetted chain (HNC) theory. ${ }^{28,29}$ However, similarly to other authors, here we shall use only the MSA theory as a first approximation.

Our numerical procedure involves the numerical solution of Eq. (35), together with the equations defining $\Phi(r)$, using a standard Picard iteration method. The grid size that we employed was $0.025 d$. When the norm of the difference vector between the $k$ th and $(k+1)$ th iterates of the $g_{i}(r)$ was less than $10^{-7}$, the iteration was deemed to have converged.

\section{RESULTS}

We refer again to the geometry that is displayed in Fig. 1. Density profiles for $\Delta=d$ and $\sigma_{2}=-\sigma_{1}$ are plotted in Figs. 2 and 3. Figure 2 shows the results at a low value of $\sigma_{2}$, while results for a relatively large value of $\sigma_{2}$ are given in Fig. 3. The density functional (DF) curves were obtained by using the bulk densities provided by the simulations. This makes possible a consistent comparison between DF and MC results. At low charge, the concentration of the ions in the membrane is low. However, at high charge, quite large number of ions are absorbed into the membrane. The anion and the cation profiles in the membrane are nearly symmetric. Thus, even at large $\sigma_{2}$, the net charge integrated over the thickness of the membrane is quite small. Outside the membrane, the values of the charge profiles near the edge of the membrane increases as the magnitude of $\sigma_{2}$ increases. However, the values are nowhere near as large as the contact values for a double layer, where the contact value increases as the square of the surface charge density. This is partly because, in contrast to the double layer, the membrane surface can be penetrated. The other reason is that the attractive
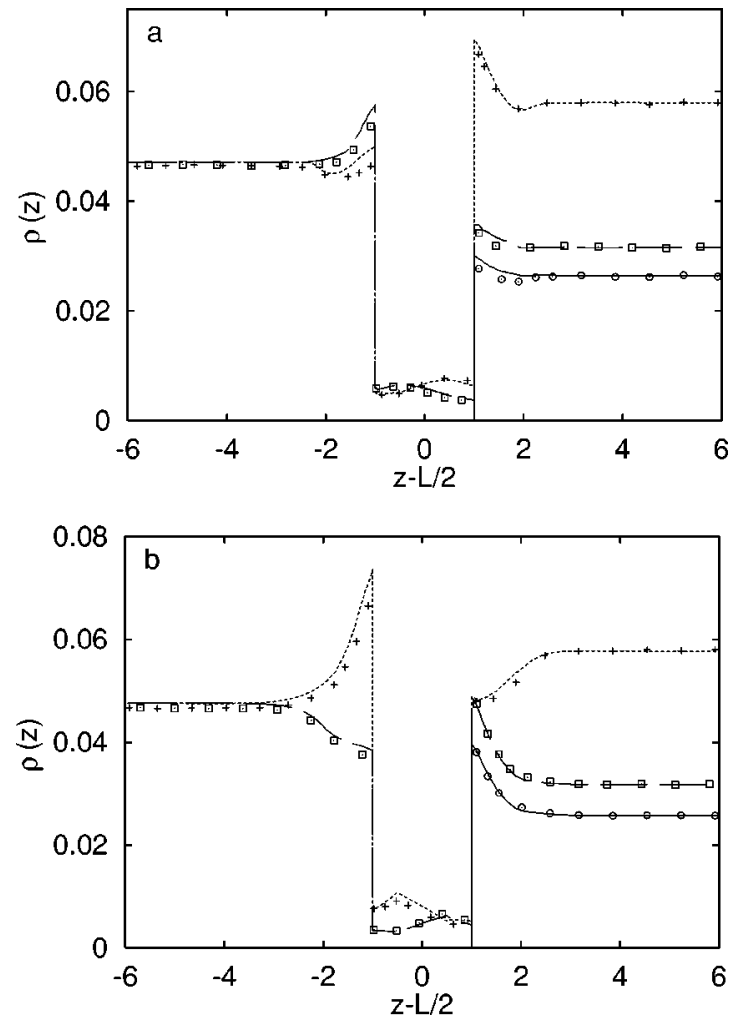

FIG. 2. Density profiles of the ions near a charged membrane for $\Delta=d$ and $B=0.1$. In parts $a$ and $b, \sigma_{2}^{*}=-\sigma_{1}^{*}=0.02778$ and -0.05556 , respectively. The bulk densities on the RHS in parts $a$ and $b$ are 0.02643 and 0.02810 for the nonpenetrating cations (solid curves and circles), 0.03151 and 0.03531 for the penetrating cations (dashed curves and squares), and 0.05794 and 0.06341 for the anions (dotted curves and plus signs), respectively. The curves and the symbols represent the DF and the MC results, respectively.

effect of the charged sheet is moderated by the other, oppositely charged sheet of the membrane; i.e., together the two, oppositely charged sheets of the membrane form a dipolelike construction in contrast to the single charged wall of the double layer.

Density profiles for the case $\Delta=0$ are shown in Fig. 4 . In this case, only the counterions (anions) can approach the membrane. The anion profile is nearly symmetric about the membrane. Only the anions have an appreciable density inside the membrane.

The DF density profiles coincide with the simulation results rather well. The magnitudes of the peaks of the DF profiles near the membrane are slightly larger than the $\mathrm{MC}$ data but otherwise the agreement is quite satisfactory. As a consequence, the DF theory can be used to investigate the behavior of the membrane in more detail. For convenience, in the following DF calculations, the densities of the penetrating and non-penetrating cations were given the same value.

In Fig. 5, the relationship between the potential and the charge on the membrane, as obtained from the DF theory, is displayed for $\Delta=d$. Even though $\sigma_{2}=-\sigma_{1}, V_{2} \neq-V_{1}$, as one would expect. At low densities or small values of $B$ and small $\sigma$, the relation between $V$ and $\sigma$ is nearly linear. However, for $B=0.1$ (the more permeable membrane) and large density, the $\sigma-V$ relationship becomes nonlinear at large 

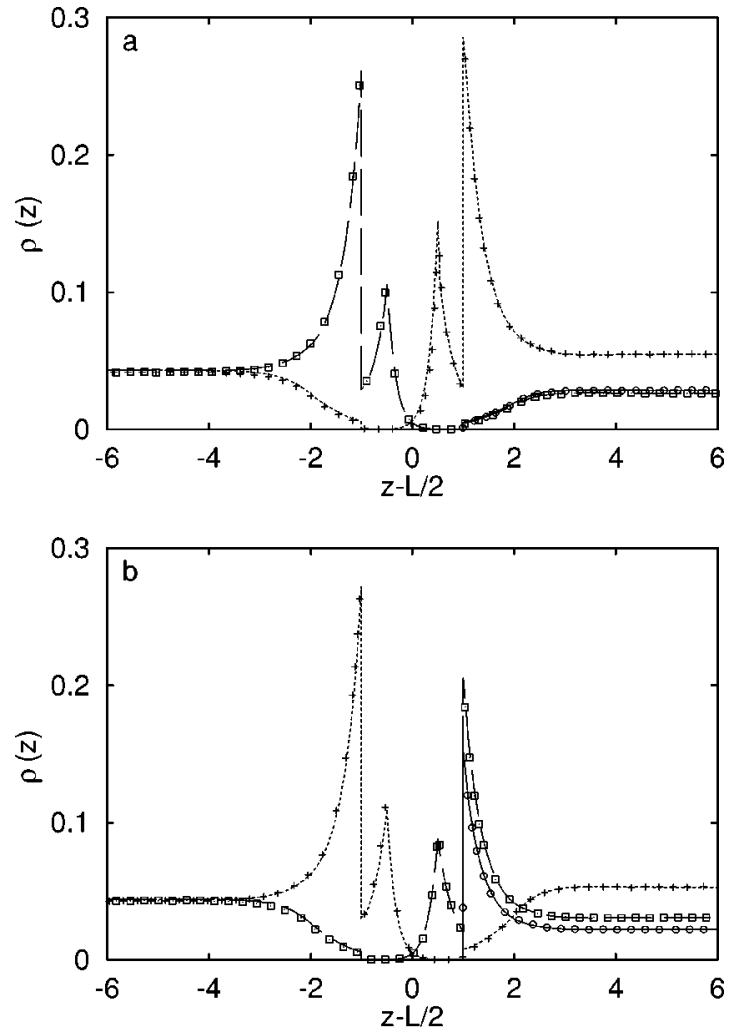

FIG. 3. Density profiles of the ions near a charged membrane for $\Delta=d$ and $B=0.1$. In parts $a$ and $b, \sigma_{2}^{*}=-\sigma_{1}^{*}=0.444$ and -0.444 , respectively. The bulk densities on the RHS in parts $a$ and $b$ are 0.03054 and 0.02217 for the nonpenetrating cations, 0.02852 and 0.03072 for the penetrating cations, and 0.05905 and 0.05290 for the anions, respectively. The meaning of the curves and the symbols is the same as in Fig. 2.

potentials (solid curve in Fig. 5). There is even a cross over of the curves at large negative values of $V_{2}$ (large values of $\left.V_{1}\right)$. This is due to the strong absorption of charge within the membrane at large $V_{2}$ as explained in Figs. 6(a)-6(c) and Figs. 7(a)-7(b). Figure 6(a) shows the density profiles for various values of the potential in the situation represented by the solid curve in Fig. 5. It can be seen that absorption peaks appear in the membrane. As the potential increases, these peaks become strongly asymmetric and much higher than the

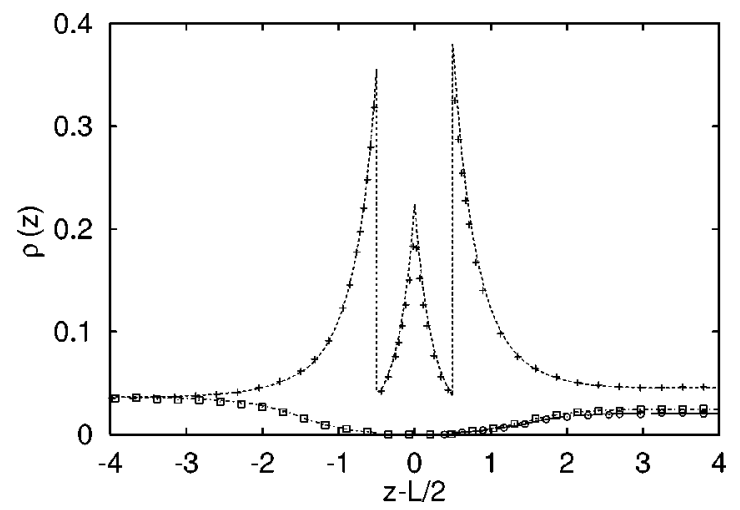

FIG. 4. Density profiles of the ions near a charged membrane for $\Delta=0$ and $B=0.1$ for $\sigma^{*}=0.1$. The bulk densities on the RHS are 0.02075 for the nonpenetrating cations, 0.02450 for the penetrating cations, and 0.04526 for the anions. The meaning of the curves and the symbols is the same as in Fig. 2.

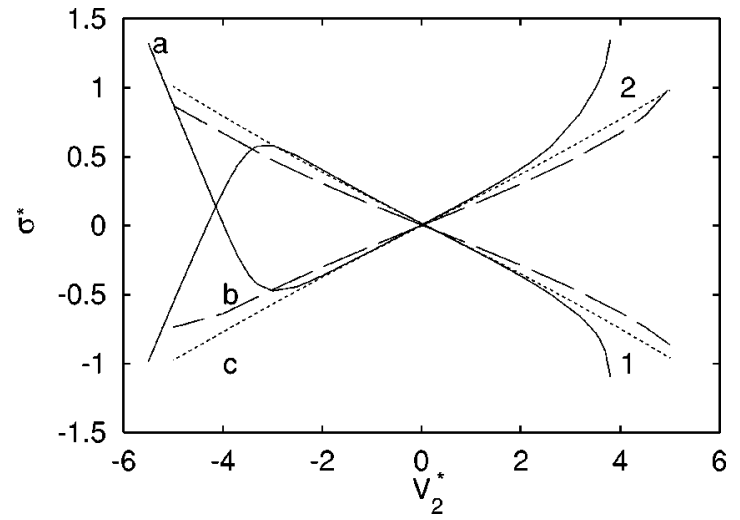

FIG. 5. $V^{*}$ as a function of $\sigma^{*}$ for $\Delta=d$. Curve $a$ (solid) and $b$ (dashed) are for $B=0.1$ whereas curve $c$ (dotted) is for $B=0.001$. For curves $a$ and $c$, the RHS bulk densities for the anions, the nonpenetrating cations, and the penetrating cations are $0.05,0.025$, and 0.025 , respectively. For curve $b$, these values are $0.005,0.0025$, and 0.0025 , respectively. The numbers near each set of curves indicate whether the set is for $V_{1}$ or $V_{2}$ as a function of $\sigma$.
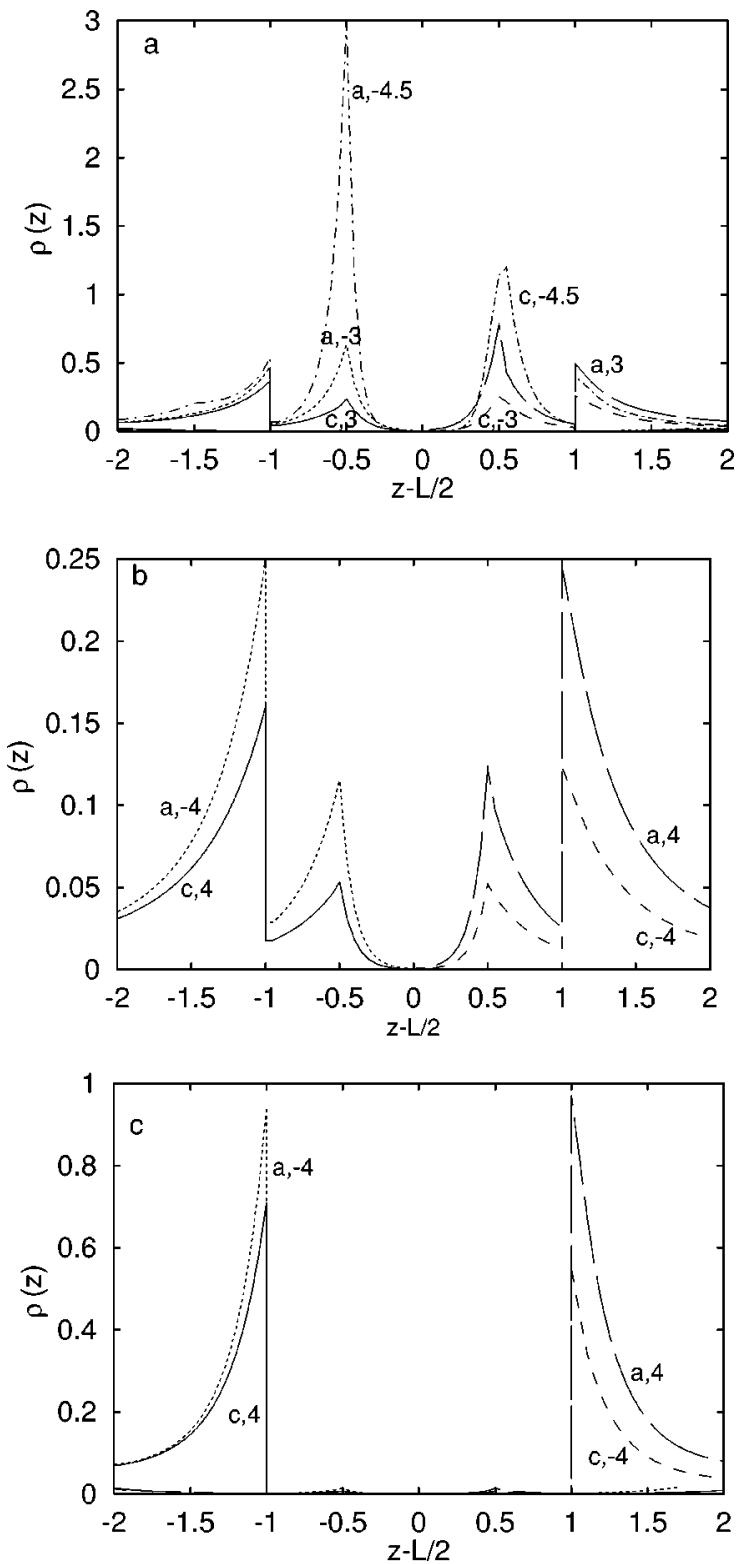

FIG. 6. Density profiles of the ions near a charged membrane for $\Delta=d$ at various values of the potential. Parts $a, b$, and $c$ correspond to curves $a, b$, and $c$ of Fig. 5. The numbers near the curves give the value of $V_{2}^{*}=-V_{1}^{*}$. 

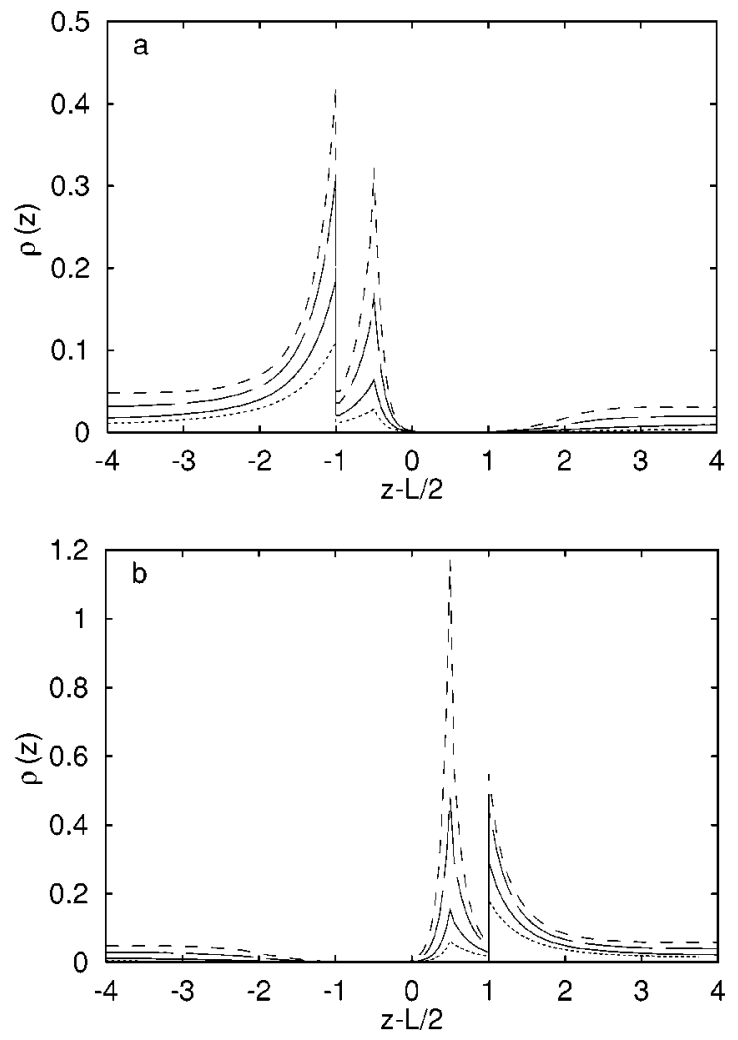

FIG. 7. Density profiles of the cations (part $a$ ) and the anions (part $b$ ) near a charged membrane for $\Delta=d, B=0.1$, and $V_{2}^{*}=-V_{1}^{*}=3$. From the top the four curves are for RHS bulk anion densities of 0.06, 0.04, 0.02, and 0.01 . The RHS bulk densities for the penetrating and nonpenetrating cations are equal.

peaks outside the membrane. This causes the anomalous behavior of the $V-\sigma$ curve. When the membrane is less permeable $[B=0.001$, see Fig. $6(\mathrm{~b})]$ or the bulk density is low $\left[\rho^{*}=0.01\right.$, see Fig. 6(c)], this behavior is absent, at least for the charges considered.

Cation [Fig. 7(a)] and anion [Fig. 7(b)] profiles are given for different values of the bulk density at a fixed value of the potential and for $\Delta=d$. The absorption of ions in the membrane increases as the total bulk density increases. Comparing the cation and anion profiles, it can be seen that the absorption profiles become more asymmetric and larger than the outside peaks as the density increases. This explains why the nonlinearity in the $V-\sigma$ relation appears at higher densities.

\section{SUMMARY}

In order to obtain some idea of the behavior of a membrane, we have constructed a very simple model membrane. The passage of ions through a biological membrane occurs in channels. Our model membrane does not have this property. For simplicity, we have assumed that the penetration of those ions that can pass through the membrane is uniform.

Density functional theory has been adapted to this model. Computations have been made for the model membrane using DF theory and MC simulations. The DF results agree quite well with the simulations.
For the more permeable membrane, there is a large absorption of charge inside the membrane when the potential on the membrane is large or when the ionic densities are large. This can result in an unusual charge-potential relationship. These potentials at which this occurs may well be unphysically large. Nevertheless, one of our purposes here is to explore the behavior of this model membrane under extreme conditions.

\section{ACKNOWLEDGMENTS}

The work of D.B., D.H., and R.R. was supported in part by the National Science Foundation (Grants CHE96-01971 and CHE98-13729), by the donors of the Petroleum Research Fund, administered by the American Chemical Society (Grant No. ACS-PRF 31573-AC9), and by a NATO High Technology Collaborative Research Grant (Grant No. HTECH, CRG 972915).

${ }^{1}$ J. G. Powles, S. Murad, and P. V. Ravi, Chem. Phys. Lett. 188, 21 (1992); S. Murad and J. G. Powles, J. Chem. Phys. 99, 7271 (1993); J. G. Powles and M. Pagoda, Mol. Phys. 78, 757 (1993).

${ }^{2}$ G. Stell and C. G. Joslin, Biophys. J. 50, 855 (1986).

${ }^{3}$ L. H. Bartlett and R. A. Kromhout, Bull. Math. Biophys. 14, 385 (1952).

${ }^{4}$ Y. Zhun and G. Stell, J. Chem. Phys. 89, 7010 (1988); 89, 7020 (1988).

${ }^{5}$ M. Calleja and G. Rickayzen, Mol. Phys. 79, 809 (1993); N. Margaritis and G. Rickaysen, ibid. 90, 189 (1997).

${ }^{6}$ D. Henderson, A. D. Trokhymchuk, and O. Pizio, Chem. Phys. Lett. 245, 615 (1995).

${ }^{7}$ B. Hille, Ionic Channels of Excitable Membranes, 2nd ed. (Sinauer, Sunderland, MA, 1992).

${ }^{8}$ D. Agins, Proc. Natl. Acad. Sci. USA 57, 1232 (1967).

${ }^{9} \mathrm{~K}$. S. Cole, Membranes, Ions, and Impulses (University of California, Los Angeles, 1968).

${ }^{10}$ W. J. Ellis, Mol. Phys. 82, 973 (1994).

${ }^{11}$ S. L. Carnie and D. Y. C. Chan, J. Chem. Phys. 73, 2949 (1980); L. Blum and D. Henderson, ibid. 74, 1902 (1981).

${ }^{12}$ D. Henderson and M. Lozada-Cassou, J. Colloid Interface Sci. 114, 180 (1986). A comparison of this theory with experiment can be found in Fig. 13.10 of J. Israelachvili, Intermolecular and Interface Forces, 2nd ed. (Academic, London, 1992).

${ }^{13}$ G. M. Torrie and J. P. Valleau, J. Chem. Phys. 73, 5807 (1980).

${ }^{14}$ Y. Rosenfeld, Phys. Rev. Lett. 63, 980 (1989).

${ }^{15}$ T. Biben, J. P. Hansen, and Y. Rosenfeld, Phys. Rev. E 57, R3727 (1994).

${ }^{16}$ E. Kierlik and M. L. Rosinberg, Phys. Rev. A 42, 3382 (1990).

${ }^{17}$ E. Kierlik and M. L. Rosinberg, Phys. Rev. A 44, 5025 (1991).

${ }^{18}$ Z. Tang, L. Mier y Teran, H. T. Davis, L. E. Scriven, and H. S. White, Mol. Phys. 71, 369 (1990).

${ }^{19}$ C. N. Patra and K. Ghosh, Phys. Rev. E 47, 4088 (1993).

${ }^{20}$ M. Lozada-Cassou, J. Chem. Phys. 80, 3344 (1984); Fundamentals of Inhomogeneous Fluids, edited by D. Henderson (Academic, New York, 1992), Chap. 8.

${ }^{21}$ D. Boda, K. Y. Chan, and D. Henderson, J. Chem. Phys. 109, 7362 (1998).

${ }^{22}$ R. Evans and T. J. Sluckin, Mol. Phys. 40, 413 (1980).

${ }^{23}$ G. Gouy, J. Phys. (France) 9, 457 (1910).

${ }^{24}$ D. L. Chapman, Philos. Mag. 25, 475 (1913).

${ }^{25}$ E. Waisman and J. L. Lebowitz, J. Chem. Phys. 52, 430 (1970); 56, 3093 (1972).

${ }^{26}$ L. Blum, Theoretical Chemistry: Advances and Perspectives, edited by D. Henderson (Academic, New York, 1980), Vol. 5, Chap. 1.

${ }^{27}$ J. S. Høye, J. L. Lebowitz, and G. Stell, J. Chem. Phys. 61, 3252 (1974).

${ }^{28}$ J. C. Rasaiah and H. L. Friedman, J. Chem. Phys. 48, 2742 (1968); 50, 3965 (1969)

${ }^{29}$ J. C. Rasaiah, Chem. Phys. Lett. 7, 260 (1970); J. Chem. Phys. 52, 704 (1970) 